\title{
Ärztetag debattiert über Praxisnetze
}

\author{
Der Deutsche Ärztetag will die Bandbreite ärztlicher Kooperationen \\ diskutieren. Das Versorgungsgesetz hat dabei die Karten für Praxis- \\ netze neu gemischt.
}

Der Vertragsarzt der Zukunft ist kein Einzelkämpfer - dieses berufspolitische Mantra schafft es auf die Bühne des 115. Deutschen Ärztetages in Nürnberg. In einem eigenen Themenblock werden sich die Delegierten mit kooperativen Versorgungsformen beschäftigen. „Wir wollen Handlungsoptionen diskutieren und aufzeigen, sowie Problembewusstsein bei den Ärzten schaffen, sagt Dr. Max Kaplan, Präsident der Bayerischen Ärztekammer. Ein Schwerpunkt werden dabei Praxisnetze bilden. Beispielhaft wird der Hausarzt Dr. Veit Wambach als Vorstand der Agentur deutscher Ärztenetze referieren. Wambach leitet seit vielen Jahren das Praxisnetz Nürnberg-Nord, aus dem später das Netz „Qualität und Effizienz -

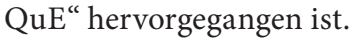

Praxisnetze haben viele Konjunkturen erlebt - und sind nicht selten mit Erwartungen der Politik überfrachtet worden: Sie sollen Heilmittel beispielsweise gegen Koordinationsdefizite oder mangelnde Patienten- und Qualitätsorientierung sein. Bislang kennt das Sozialgesetzbuch V Vertragsärzte als Teilnehmer an Strukturverträgen (Paragraf 73 a) oder an Modellvorhaben (Paragraf 63ff.). Der Begriff Praxisnetz kam bis dato nicht vor.

\section{Infobox 1} tig. den ist. konzepten auftreten.

\section{Honorarbudget}

für Praxisnetze denkbar

\section{Netzagentur will Lobbyarbeit für Verbünde machen}

Wenn Praxisnetze ein Motor der regional angepassten Versorgung sein sollen, dann ist eine professionelle Begleitung der Netze nö-

Dies ist die Kernidee der Agentur deutscher Ärztenetze, die im vergangenen Jahr von zunächst 14 Mitgliedern gegründet wor-

Die Netzagentur versteht sich als politische Interessenvertretung für über 400 Ärztenetze in Deutschland. Sie will die Mitglieder bei der Professionalisierung unterstützen und als Dienstleister bei Vertragsund Versorgungs-

Die Netzagentur will sich dafür einsetzen, dass Ärztenetze als Leistungserbringer im SGB $V$ anerkannt werden und Netze einen regionalen Teilsicherstellungsauftrag erhalten.

Das hat sich mit dem Versorgungsstrukturgesetz geändert. Nach dem neuen Paragrafen 87b SGB V können Netze schrittweise in die vertragsärztliche Versorgung integriert werden. Danach kann die KV einem Praxisnetz ein eigenes

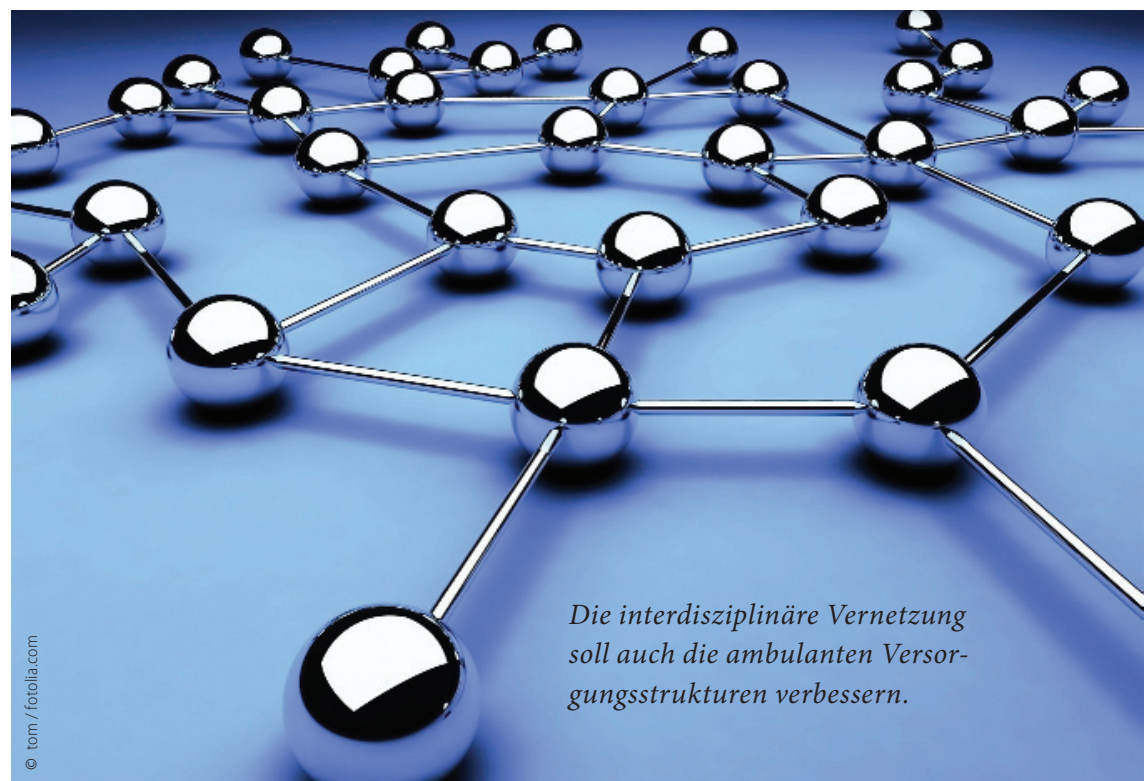

\section{Infobox 2}

So steht es im Gesetz

Paragraf 87b SGB V

- Absatz 2: Der Verteilungsmaßstab hat der kooperativen Behandlung von Patienten in dafür gebildeten Versorgungsformen angemessen Rechnung zu tragen; dabei können auch gesonderte Vergütungsregelungen für vernetzte Praxen (...) vorgesehen werden, soweit dies einer Verbesserung der ambulanten Versorgung dient und das Praxisnetz von der $\mathrm{KV}$ anerkannt wird.

- Absatz 4: Die KBV hat Vorgaben zur Festlegung und Anpassung des Vergütungsvolumens für die haus- und fachärztliche Versorgung (...) sowie Kriterien und Qualitätsanforderungen für die Anerkennung besonders förderungswürdiger Praxisnetze als Rahmenvorgabe für Richtlinien der KVen, insbesondere zu Versorgungszielen, im Einvernehmen mit dem GKVSpitzenverband zu bestimmen.

Honorarbudget als Teil der Gesamtvergütung zuweisen. Das Praxisnetz gibt die Mittel dann nach einem Verteilungsschlüssel an die Netzteilnehmer weiter. Dies eröffnet Netzen die Option, sowohl im Selektiv - wie auch im Kollektivvertragssystem tätig werden zu können.

Klar im Fokus des Gesetzgebers sind dabei „Zusammenschlüsse von Vertragsärzten verschiedener Fachrichtungen zur interdisziplinären (...) Betreuung, die die ambulanten Versorgungsstrukturen verbessern“. Die vielfältigen fachgruppenspezifischen Netze fallen vor diesem Hintergrund durch das Raster des Gesetzgebers.

Die KBV hat nun die Aufgabe, bundesweit gültige Kriterien für die Förderfähigkeit von Netzen zu entwickeln. Ob am Ende dieser Entwicklung tatsächlich mehr regionale Versorgungsmodelle entstehen, ist ungewiss. Der Deutsche Ärztetag hat das Thema Kooperation mitten in einer Umbruchsituation für Netze auf seine Agenda gehoben.

(Florian Staeck) 